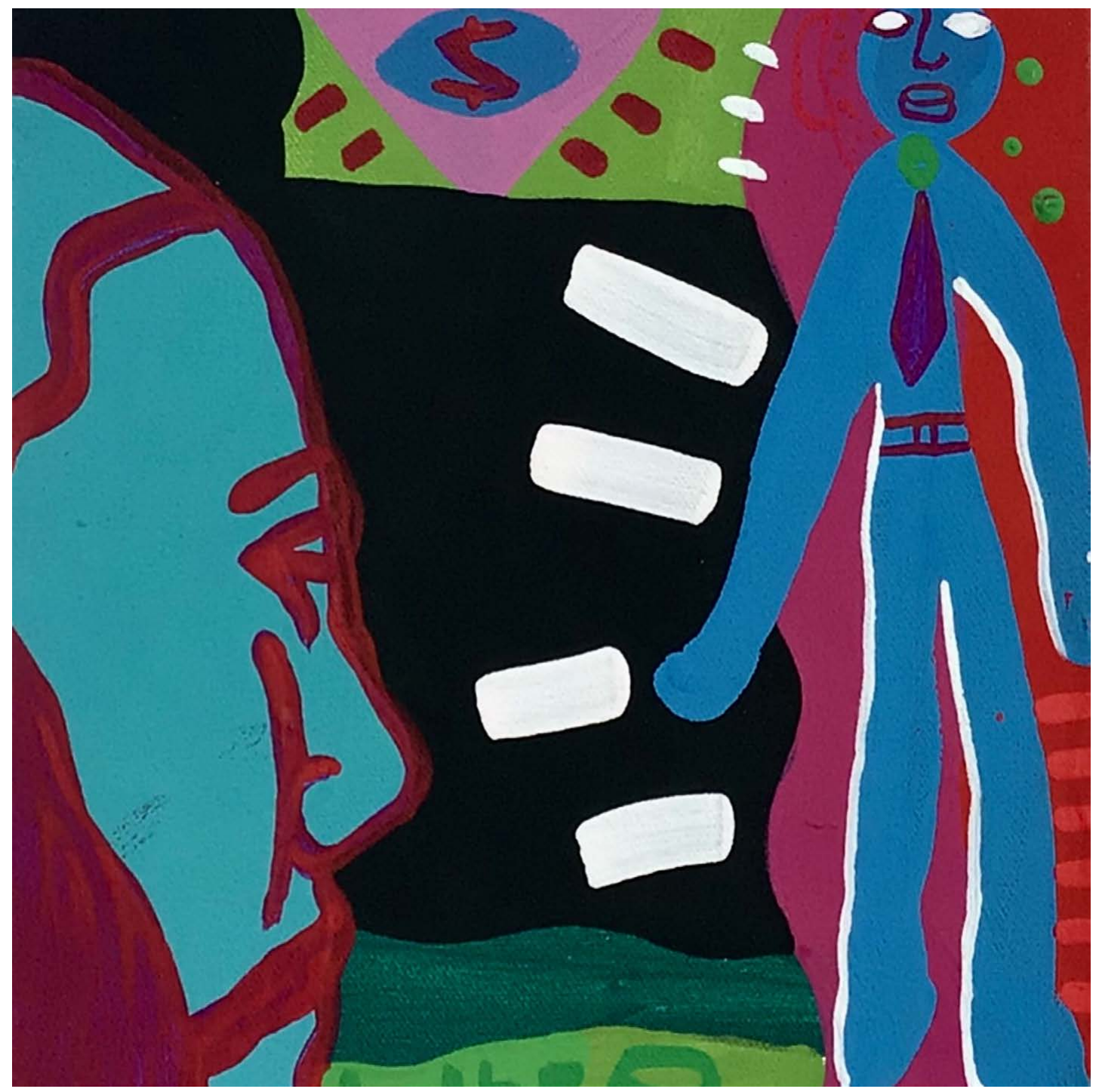

Artista invitado

Fabio Andrés Arboleda Mendoza $\$$

De la serie neWeed

Acrílico sobre lienzo

$20 \times 20 \mathrm{~cm}$

2020

Medellín 


\title{
Análisis de género en los contextos de violencia organizada. Una mirada sociológica ${ }^{*}$
}

\author{
Anabel Garrido Ortolá (España)**
}

\section{Resumen}

Este artículo realiza una reflexión teórica acerca de cómo se estructuran las dinámicas de género en los contextos de violencia organizada. El objetivo es acercarse a un marco explicativo que pueda ofrecer un enfoque feminista y sociológico sobre el género en la organización social de la violencia. Las preguntas que subyacen son: ¿cómo ha sido la participación de las mujeres en la guerra? y ¿Cómo se debería enfocar el análisis de género en la violencia organizada? Para ello se abordan tres problemas universales que se dan como dinámicas sociales: i) la estratificación del género: la organización social de la violencia; ii) las violencias específicas contra las mujeres en los conflictos armados; y iii) la reificación de los roles de género: violencia implícita. Estas dinámicas muestran cómo se refuerza la violencia contra las mujeres en estos contextos, no solo por la violencia específica

[ 80 ] contra sus cuerpos, sino también por la violencia ejercida a través de la reificación de los roles de género.

\section{Palabras clave}

Teoría Política; Conflicto Armado; Guerra; Subjetividades Políticas; Feminismo; Género.

Fecha de recepción: noviembre de 2020

- Fecha de aprobación: agosto de 2020

\section{Cómo citar este artículo}

Garrido Ortolá, Anabel. (2021). Análisis de género en los contextos de violencia organizada. Una mirada sociológica. Estudios Políticos (Universidad de Antioquia), 62, pp. 80-104. https://doi.org/10.17533/udea.espo.n62a04

\footnotetext{
*Artículo derivado del proceso de investigación del Doctorado en Sociología y Antropología, Universidad Complutense de Madrid, Ni víctimas, ni victimarias. Análisis de las narrativas de género en torno al conflicto y postconflicto armado en Colombia (2017), bajo la dirección de la profesora María Luisa Revilla Blanco.

${ }^{* *}$ Licenciada en Ciencias Políticas y de la Administración. Máster en Desarrollo y Ayuda Internacional. Máster en Relaciones Internacionales. Doctora en Sociología y Antropología. Profesora Asociada de la Universidad Complutense de Madrid, España. Correo electrónico: angarrid@ucm.es - Orcid: 00000002-5379-2852 - Google Scholar: https://scholar.google.es/citations?user=7WgoLnYAAAAJ\&hl=es
} 


\title{
Gender Analysis in the Contexts of Organized Violence. A Sociological Perspective
}

\begin{abstract}
This article makes a theoretical reflection on how gender dynamics are structured in the contexts of armed conflict. The objective is to approach an explanatory framework that can offer a feminist and sociological approach to gender in the social organization of violence. The underlying question is, how has the participation of women in the war been? How should gender analysis be approached in organized violence? To this aim, three universal problems that take place as social dynamics are addressed: 1) The stratification of gender: the social organization of violence; 2) Specific violence against women in armed conflicts; and 3) The reification of gender roles: implicit violence. These dynamics show how violence against women is reinforced in these contexts, not only because of the specific violence against their bodies, but also because of the violence exercised through the reification of gender roles.
\end{abstract}

\section{Keywords}

Political Theory; Armed Conflict; War; Political Subjectivities; Feminism; Gender. 


\section{Introducción}

Los contextos de violencia organizada, como la guerra o los conflictos armados, han sido considerados espacios masculinos, caracterizados por la participación principalmente de hombres. En la llíada, Héctor le dice a su esposa: «vuelve a casa, ocúpate en las labores del telar y la rueca, y ordena a las esclavas que se apliquen al trabajo; y de la guerra nos cuidaremos cuantos varones nacimos en Ilión, y yo el primero» (Homero, 1996, p. 83). La guerra se conforma como el último bastión masculino, que se ha caracterizado no solo por la exclusión de las mujeres de ella, sino también por una tradicional mirada androcéntrica de los estudios de la violencia organizada.

El concepto de violencia organizada indica la necesidad de contar con estructuras y mecanismos que permitan la organización de una violencia que puede ser prolongada (Malesevic, 2020). En este sentido, el concepto permite diferenciar la violencia interpersonal de la violencia organizada. En este artículo se utiliza como concepto amplio sobre violencia, el cual adquiere medios y estructura —organizada- y que permite prolongarse en el tiempo, independientemente de que se observe como conflicto armado o guerra.

Por su parte, el concepto de conflicto armado se aborda aquí de manera equiparable con el de guerra, aun siendo conscientes de las diferencias entre ambos conceptos. Entre las definiciones tradicionales de la guerra, hay quienes se enfocan en cantidad de víctimas (Pinker, 2012). El Stockholm International Peace Research Institute (SIPRI, 2008) define la guerra como un conflicto armado mayor - major armed conflicto- que cuente con una disputa por el territorio o el gobierno con uso de armamento, siendo una de las partes el Gobierno y contando con un número de bajas no menor a un millar al año. Otra definición que permite diferenciar ambos conceptos indica que los conflictos armados se caracterizan por ser internos (Fisas, 1987). De este modo, independientemente de su intensidad o sus fronteras, ambos conceptos se utilizan aquí como equiparables, ya que permite establecer un marco de actuación de la violencia organizada.

Diversos estudios sobre las dinámicas de género en la guerra han mostrado resultados diferenciados por sus perspectivas analíticas. Desde el esencialismo biológico - que explica la participación de hombres y mujeres en el mundo desde la condición sexuada-, así como los enfoques culturalistas que indican cómo la construcción sexuada de la guerra se determina por 
la construcción cultural del sistema de dominación masculina. Si bien es cierto que en los escenarios de conflicto armado el sistema de dominación masculina se refuerza y acentúa (Cockburn, 2007; Magallón, 1998; Mesa, 2012), esta no explica por sí misma la estratificación universal de género en la guerra (Malesevic, 2010).

La guerra se constituye como un escenario de masculinidad hegemónica que ha sido principalmente escenario exclusivo $-\mathrm{y}$ excluyente- de hombres. El concepto de masculinidad se aborda como aquella establecida en el discurso hegemónico binario. No obstante, cabe señalar que no existe una única masculinidad sino varias. Por ejemplo, se distinguen cuatro tipos: masculinidad hegemonial, subordinada, cómplice y marginalizada (Connell, 1995). En este artículo se aborda el concepto de masculinidad hegemónica como aquel referido por masculinidad hegemonial, entendiéndose como «la configuración de práctica genérica que encarna la respuesta corriente aceptada al problema de legitimidad del patriarcado» (p.39).

Este círculo cerrado establece un conjunto de elementos significativos de poder y prestigio, alejando a las mujeres constituidas en la periferia de este centro masculino (Brownmiller, 1975; Osborne, 2009), garantizando «la posición dominante de los hombres y la subordinación de las mujeres» (Connell, 1995, p. 39). Para abordar esta premisa, cabe preguntarse: ¿Cómo se organiza la violencia? y ¿cuál es el papel otorgado al género en esta organización social?

Es necesario, por tanto, recoger un marco sociohistórico que permita estudiar los procesos sociales de organización social de la guerra y su configuración de género. Para acometer esta revisión teórica se asume las tesis de Max Weber (1964), Charles Tilly (1990) y Sinisa Malesevic (2010), a partir de un enfoque feminista. Este encuadre permite establecer la mirada sobre los conflictos armados y preguntar cuál es el papel de las mujeres en los conflictos actuales.

Desde una mirada empírica que permita observa la participación de las mujeres, la información indica que ellas han participado tan activamente como los hombres. Su participación ha sido como víctimas sobrevivientes de la violencia, victimarias enroladas en grupos armados o sujetas políticas, como activistas por la construcción de paz en el territorio. Estas tipologías de participación permiten centrar el análisis en tres categorías sociales para su 
sistematización: víctimas, victimarias y sujetas políticas. Es a través de estas tres categorías que se abordará la participación de ellas.

Es necesario señalar que las categorías de análisis son conceptos que permiten acercarse al problema a través de la categorización; no obstante, aunque se aborden las categorías como conceptos delimitados, la interrelación entre las distintas categorías es continua, pudiendo observarse mujeres en distintas posiciones, tales como víctimas, que se erigen como sujetas políticas, incluso mujeres que han estado en las tres categorías - excombatientes que han sido víctimas y, a su vez, sujetas políticas en su reinserción a la vida civil-. Por eso es necesario concebir las distintas categorías de análisis como situaciones vivenciales mutables y transformables, y no reificadas en torno a la «condición» de género.

La diversa participación de las mujeres en los contextos de conflictos armados conlleva el siguiente cuestionamiento: ¿cómo enfocar un análisis de género en los contextos de conflicto armado? Para dar respuesta a esta cuestión, este artículo incursiona en tres problemas universales que se dan como dinámicas sociales y que conforman los condicionamientos del género en los contextos de violencia organizada: i) la estratificación del género: la

[ 84 ] organización social de la violencia; ii) las violencias específicas contra las mujeres en los conflictos armados; y iii) la reificación de los roles de género: violencia implícita.

\section{La estratificación del género: la organización social de la violencia organizada}

La creación del Estado nacional se configura a través de la guerra, condicionado por el eje coerción-Estado-dominación (Tilly, 1990, p. 44). Es a través de este desarrollo histórico que se instituye la polarización del género que se refuerza ante la expansión de la civilización y del poder del Estado nacional (Malesevic, 2010).

Al realizar una revisión histórica, la constitución social revela que la conformación de bandas nómadas se identificaba por la carencia de organización, por la alta igualdad interna y por poseer un carácter no violento (Service, 1978; Fry, 2007; Malesevic, 2010). Estas organizaciones sociales evidencian la falencia de un sistema patriarcal como forma específica de dominación masculina sobre las mujeres. La estratificación del 
género no se daba en las organizaciones previas a las sedentarias, es decir, en las organizaciones tribales basadas en el parentesco. Esto es debido, en parte, a la ordenación social basada en un liderazgo frágil, sin la capacidad de un poder coercitivo (Service, 1978; De Waal, 2005; Malesevic, 2010). Es «con el desarrollo de los cacicazgos y estados prístinos» que el orden social se establece como jerárquico y se dé la estratificación de género en la configuración social, emergiendo ambos hechos a la par (Malesevic, 2010, p. 298).

No obstante, aun concibiendo la dependencia existente entre ambos hechos, es difícil probar la causalidad de ellos. Del mismo modo, el mayor desarrollo de la organización social permitió la generación de diversas formas de jerarquía social (Malesevic, 2010). Esta estratificación social se genera en torno a un cierre social que, mediante elementos coercitivos y capitalistas, mantiene la consecución de las jerarquías en las cuales hay ascenso de élites políticas (Weber, 1964; Tilly, 1985; Mann, 1986). Partiendo de esta base, los condicionamientos que llevan a la ausencia de las mujeres en los campos de batalla se derivan de los mecanismos de construcción en la organización social, mediante la división sexual del trabajo en dos esferas: la civil y la militar (Malesevic, 2010). Si bien las mujeres han sido excluidas de la esfera militar, también lo han sido aquellos hombres que no han conseguido entrar, siendo nombrados «mujeres»: "quien no resiste las pruebas de guerra es una "mujer", es decir, está excluido del séquito» (Weber, 1964, p. 200). Sin embargo, la capacidad de las mujeres para participar como victimarias en la guerra ha sido muchas veces comprobada en distintos momentos históricos; por tanto, esta exclusión está condicionada por la configuración del orden social y no por cuestiones biologicistas.

Llegados a este punto cabe preguntarse: ¿cuál es el elemento diferenciador que estructura esta organización social? La división sexual del trabajo es el fenómeno social que ha servido como explicación para el mantenimiento de estas jerarquías sociales, pero ¿cómo opera en la guerra? Esta división sexual se establece en la capacidad biológica de reproducirse, las mujeres son protegidas de la guerra por ser el único género capaz de concebir y alimentar a nuevas generaciones de guerreros o trabajadores; por lo tanto, la exclusión de las mujeres de la esfera militar no es producto de su incapacidad de lucha, sino por su capacidad de crear vida (Malesevic, 2010). 
La guerra configura una organización social que se instituye a través de la combinación de elementos coercitivos y materiales, a la vez de conllevar un mayor prestigio social (Tilly, 1990). Esto es debido a que «aquellos que ejercían la coerción, que tuvieron parte principal en la creación de los Estados nacionales, se valieron para sus propios fines de manipuladores de capitales, cuyas actividades generaron ciudades» (Tilly, 1990, pp. 40). En esta línea, son principalmente los guerreros quienes adquirieron una posición privilegiada en la sociedad, debido a su control de «todos los medios de coerción» (Malesevic, 2010, p. 297).

Lo que inicia como una necesidad de división sexual para la organización social acaba convirtiéndose en una jerarquía sexuada, en la cual la figura del guerrero se sitúa en la cúspide social, cerrando el acceso a otros grupos sociales (Weber, 1964), principalmente al género femenino. Así pues, la exclusión de las mujeres de la esfera militar se establece mediante dos procesos conexos: la «burocratización acumulativa de la violencia» y la «ideologización centrífuga de los roles de género» (Malesevic, 2010, p. 299).

\subsection{La conformación del ethos guerrero}

La «burocratización acumulativa de la violencia» alude a la posición privilegiada que deriva de la participación coercitiva de la guerra, construida respecto de la disposición material de recursos económicos de los guerreros que les confiere un estatus en la organización social (Malesevic, 2010). Este estatus del ethos guerrero se configura en torno a la autoridad de estos en la sociedad; así pues, las jerarquías sociales se basan «primeramente de los fuertes y después de la gente con experiencia, por consiguiente, de los hombres respecto de las mujeres y niños, de los aptos para la guerra y para el trabajo respecto de los ineptos, de los adultos respecto de los niños, de los viejos sobre los jóvenes» (Weber, 1964, p. 291). De esta definición se desprende la configuración de las jerarquías que establece al hombre guerrero y fuerte, apto para la guerra y el trabajo, con mayor autoridad social ante las mujeres.

La participación de las mujeres en la esfera militar puede no solo «perturbar la relación simétrica de obreros y trabajadores de apoyo a los combatientes, sino que también socavaría el carácter de género de las relaciones de poder dentro de la organización social», suponiendo un 
perjuicio para tal (Malesevic, 2010, p. 301). Por lo tanto, es a través de la vertebración de la organización social en la conformación coercitiva de los Estados que se instituye la estratificación de género mediante un eje entre género, militarismo y organización social (Malesevic, 2010).

En la jerarquización social se genera un «cierre» que conduce al «mantenimiento de una alta calidad y por tanto (eventualmente) del prestigio y de las probabilidades inherentes, de honor y (eventualmente) de ganancia» (Weber, 1964, p. 37). Este "cierre monopolista» establece una esfera militar conformada por prestigio social y material, que «con el fin de asegurar un monopolio de hecho o de derecho respecto a ciertos puestos con exclusión» de uno de los géneros - el de las mujeres- de la esfera militar (Weber, 1964, p. 276). Este «cierre monopolista» configura una fijación en torno a la comunidad guerrera, de la cual quedan excluidas las mujeres, al igual que también lo serán aquellos hombres incapaces de ser guerreros, los cuales son considerados mujeres.

\subsection{La construcción ideológica del patriarcado}

La ideologización centrífuga alude a la construcción ideológica que configura los roles asociados al género. De este modo, se construye una «identidad [que] se convierte en una herramienta con la cual las sociedades inducen a los hombres a luchar», estableciéndo «normas culturales [que] obligan a los hombres a soportar el trauma y el miedo principal, con el fin de invocar la condición de virilidad» (Goldstein, 2001, pp. 252-264). La guerra es interpretada como una prueba de virilidad, haciendo uso de la masculinidad como rol significativo de la violencia. Rechazando las visiones esencialistas sobre la participación inherente de los hombres en la violencia, la construcción social de los roles de género en la guerra se centra en el papel establecido en la organización social. Por ello, es necesario tener en cuenta la institucionalización a través de la burocratización acumulativa de la violencia y el proceso de ideologización centrífuga (Malesevic, 2010).

La ideología es entendida como un proceso universal en el cual los sujetos individuales y sociales producen y reproducen la práctica social en forma de «pensamiento-acción» (Malesevic, 2010, p. 303). Así, se instaura la estructura normativa que indica las normas culturales en función de la dicotomía del género que condiciona un fenómeno mayor: el "grupo moral» (Malesevic, 2010, p. 303). 
Asimismo, se entiende que «la moral es lo que la sociedad es, en tanto que el hombre es un ser moral solo porque él vive dentro de las sociedades establecidas» (Durkheim, 1986, pp. 202-203). La moral opera a través de la ideologización centrífuga de los roles de género al establecer cuál es la norma y estableciendo la correlación entre la masculinidad y la guerra. Si bien la burocratización de la violencia estructura las jerarquías de género, la ideologización ofrece la justificación para que operen estas jerarquías; legitimando así «el universo moral de la guerra» (Malesevic, 2010, p. 304).

De este modo, la estratificación del género se construye por un discurso socializante que instituye el tipo de participación sexuada en la guerra, la cual condiciona una socialización binaria en torno a dos esferas: militar y civil (Malesevic, 2010, p. 304). Esta segmentación marca la división sexual de la organización social, condicionando la participación en torno a un sistema patriarcal que se conforma como ideológico.

Asimismo, las características asociadas a cada una de las esferas se estructuran en los roles del género, mediante un proceso centrífugo que impregna a la esfera social. Esta «ideologización centrífuga de los roles de género» (Malesevic, 2010, p. 302) recoge el imaginario simbólico que [ 88 ] reproduce la estratificación social del género en la guerra. La ideologización patriarcal instituye la posición social sexuada, conformando condicionantes en la tipología de participación en el combate de hombres y mujeres: reificándose las categorías sociales en torno a víctimas, victimarios(as) o sujetos(as) políticos(as).

\section{Las violencias específicas contra las mujeres en los conflictos armados}

La violencia organizada como hecho social muestra un carácter cambiante que ha de analizarse también en su contexto político y social (Malesevic, 2020). El marco temporal ha condicionado el proceso de la violencia organizada, diferenciando dos momentos: las guerras como actividad propia en la conformación del Estado nacional (Tilly, 1990) y las «nuevas guerras» que surgen debido al debilitamiento del Estado, a partir de la guerra de los Balcanes (Kaldor, 1999).

Las «nuevas guerras» refuerzan, en mayor medida, el contexto diferencial en las actividades de género, tanto en sus necesidades, la 
adquisición de recursos y control sobre estos, como en el acceso al proceso de toma de decisión (PNUD, 2003; Bell y Narayanaswamy, 2003). De este modo, indistintamente del tipo de conflicto, existe la percepción de que son los hombres quienes pelean en el campo de combate y las mujeres permanecen en sus hogares de manera segura; sin embargo, esta percepción no refleja la realidad de la violencia organizada. La distinción de zonas de conflicto armado y zonas de no conflicto —estableciendo el hogar y trabajo como lugares seguros- forma parte de una narración ficticia analizada por muchas feministas (Byrne, 1996; Cockburn, 1998; Bell y Narayanaswamy, 2003; Giles y Hyndman, 2004).

Este desdibujamiento del campo de batalla propiciado por nuevos actores y espacios de combate ha condicionado un contexto de violencia mayor. La militarización de una sociedad no solo condiciona una mayor violencia, sino que esta violencia recae principalmente contra las mujeres (Mendian, 2008). Esta se evidencia principalmente a través de la violencia sexual que sufren las mujeres como una violencia casi específica. ${ }^{1}$ Esta violencia se realiza a través de sus cuerpos por medio de violaciones, mutilaciones genitales, esclavitud sexual y malos tratos, pero también por medio del desplazamiento forzado y la pobreza vinculada a la desigualdad y a una mayor situación de vulnerabilidad (Bell y Narayanaswamy, 2003; Rehn y Johnson, 2002). Dos son los mecanismos de violencia física recurrentes en el sistema patriarcal sobre el cuerpo de las mujeres: la violencia sexual y el feminicidio. ${ }^{2}$ Ambas violencias se muestran como «elementos centrales de las relaciones de poder patriarcal, por tanto, el cuestionamiento a este tipo de violencia constituye un desafío al patriarcado» (Russell y Radford, 2006, p. 671).

\subsection{La violencia sexual}

El control llevado a cabo por este sistema patriarcal en el cuerpo de las mujeres muestra la visión del cuerpo femenino como una propiedad

\footnotetext{
${ }^{1}$ Aunque se observa una mayor violencia sexual hacia las mujeres en los contextos de conflicto armado, cada vez se reportan más datos sobre las violaciones a hombres (Stemple, 2009). Este hecho muestra cómo la violación es un «mensaje de poder y apropiación pronunciado en sociedad» (Segato, 2014, p.79). De este modo se busca quebrar a los hombres produciendo «tres miedos — miedo a la feminización, miedo a la homosexualidad y miedo a la impotencia procreadora-, que pondría en cuestión el modelo hegemónico de masculinidad» (Dador, 2005, p. 15).

${ }^{2}$ El término feminicidio alude al fenómeno amplio sobre la violencia de género, el genocidio de mujeres derivado del sistema patriarcal, señalando al Estado como responsable último (Lagarde, 2006).
} 
masculina, concibiéndose como «violable» (Bocchetti, 1996, p. 78). La violencia que sufren las mujeres en los conflictos armados se convierte en una extensión del control de los cuerpos femeninos en el patriarcado (Liss, 2004, mayo 13). El cuerpo de las mujeres forma parte del campo político en la guerra, en el cual «las relaciones de poder operan sobre él [como] una presa inmediata [...]. El cuerpo, en una buena parte, está imbuido de relaciones de poder y de dominación» (Foucault, 1975, pp. 32-33). En estas relaciones de poder el cuerpo femenino es despreciado y controlado por medio del patriarcado, si bien es cierto que estos hechos se encuentran en contextos de paz, en aquellos lugares donde existe un conflicto armado la violencia hacia las mujeres se acentúa y responde a distintas razones ligadas a la guerra contra el enemigo (Villellas, 2010).

La lógica patriarcal en los escenarios de conflicto armado introduce una dinámica distintiva en la cual el cuerpo de las mujeres — del enemigoes el foco estratégico en las violaciones masivas. Estas dinámicas exhiben la incapacidad de «los conquistados» por no saber defender a sus mujeres, revelando un «mensaje intermasculino» entre los hombres, «la cara inversa de la fratía» (Osborne, 2009, p. 177).

[ 90 ] La violencia patriarcal en los contextos de conflicto armado se muestra como un «objetivo estratégico», dejando de producirse como «efecto colateral» de los conflictos armados, ${ }^{3}$ convirtiéndose así en elemento nuclear (Segato, 2014, p. 15). El cuerpo de las mujeres se convierte en centralidad estratégica y es cosificado al ser concebido como botín de guerra. El sujeto mujer, al estar cosificado, se observa como un objeto estratégico susceptible de ser violado, utilizándose como medio para la destrucción de las comunidades enemigas a través de la violencia física. Esta dinámica específica en escenarios de guerra diferencia el fenómeno al buscar otras funciones adicionales (Aafjes, 1998), tales como:

- Establecer el miedo en una población civil a través de las violaciones de las mujeres. En esta línea, no solo se devalúa a las mujeres, sino que es una afrenta contra los hombres de la población enemiga al no poder defender sus «propiedades».

\footnotetext{
${ }^{3}$ Rita Laura Segato $(2014$, p. 22) realiza un análisis sobre cómo en las nuevas guerras se incrementa la violencia contra las mujeres, mutando de daño colateral a estrategia bélica. Estas nuevas guerras refieren a la existencia de nuevos actores - paramilitares, sicarios, maras, entre otros - en contextos bélicos donde la criminalidad y la crueldad aumenta.
} 
- Es un método de limpieza étnica a través de dos vías: violar y asesinar a las mujeres para exterminar la población, o la violación de ellas para embarazarlas.

- Como arma de divulgación, entendida a partir de dos perspectivas: como estrategia de guerra para «generar odio hacia los contrarios» y como aliciente ${ }^{4}$ masculino para los soldados.

De este modo, el cuerpo de las mujeres pasa a ser considerado como parte del campo de batalla, sirviendo como arma de guerra a las fuerzas contrarias e institucionalizándose la violencia dentro del conflicto armado (Dador, 2005; Osborne, 2009; Vendrell, 2013; Villellas, 2010). Este hecho persigue distintos objetivos: por un lado, tiene una dimensión individual que corresponde al sometimiento directo sobre la víctima de la violencia sexual; y por otro lado, la violencia sexual tiene una dimensión colectiva al pretender humillar a una comunidad o población enemiga. Al considerar el cuerpo de las mujeres como propiedades masculinas, las violaciones masivas se erigen como una herramienta de humillación al enemigo, convirtiendo los cuerpos en portadores del mensaje de poder y control (Mackenzie, 2010; Villellas, 2010; Coomaraswamy, 1999).

Por ende, la violencia sexual está motivada a destruir parte del tejido social y familiar de las poblaciones enemigas, y demostrar el poder a través de los cuerpos femeninos (Mackenzie, 2010; Villellas, 2010). La humillación que acompaña estos hechos victimizantes se debe a la consideración de las mujeres como depositarias de honor de la sociedad, al ser las transmisoras de las tradiciones como educadoras y cuidadoras de las comunidades (Kandiyoti, 2000). Así, mediante las violaciones sexuales hacia las mujeres se rompe la cadena de honor. Motivo por el cual, en la mayoría de los casos, el ocultamiento de las violaciones, tanto individual como social, busca proteger el honor masculino -ya que los hombres no han sido capaces de «proteger su propiedad»- y el estigma social de las mujeres violadas que genera la ruptura del honor (Olujic, 1998; Villellas, 2010).

Aunque las violaciones han sido elementos habituales en las guerras, en la actualidad se evidencia como una «estrategia deliberada» de guerra (Kaldor, 1999), al observarse en las violaciones colectivas, los campos de

\footnotetext{
${ }^{4}$ Este caso se encuentra en las denominadas comfort women, mujeres retenidas en burdeles militares para los japoneses, utilizadas como esclavas sexuales tras la II Guerra Mundial. La cantidad de mujeres utilizadas por tal fin se establece en torno a 100000 confortadoras (Gutman y Rieff, 2003).
} 
violación, las mutilaciones sexuales o el tráfico de mujeres para trabajos forzosos o explotación sexual. Las organizaciones internacionales califican las violaciones colectivas contra las mujeres como crímenes de guerra y de lesa humanidad, regulados en el Estatuto de Roma de la Corte Penal Internacional sobre las cuestiones relativas a la violencia de género y sexual (Rehn y Johnson, 2002).

Al margen de la dificultad para conocer la cantidad real de violaciones en los conflictos armados - debido, en parte, a la impunidad y al estigma de las mujeres violadas para denunciar-, los datos recogidos, aunque sesgados, muestran una amplia dimensión del problema (véase tabla 1).

Tabla 1. Mujeres-niñas violadas en contextos de guerra recientes.

\begin{tabular}{|l|c|}
\hline \multicolumn{1}{|c|}{ Países } & $\begin{array}{c}\text { Cantidad de mujeres-niñas } \\
\text { violadas }\end{array}$ \\
\hline Ruanda 1994 & 250000 \\
\hline Sierra Leona (1991-2002) & 60000 \\
\hline Liberia (1989-2003) & 40000 \\
\hline Ex Yugoslavia (1995-1999) & 60000 \\
\hline República Democrática del Congo ${ }^{5}$ (desde 1996) & 200000 \\
\hline
\end{tabular}

Fuente: elaboración propia a partir de Organization of African Unity (2000); Physicians for Human Rights (2002); Manuela Mesa (2012).

\subsection{Feminicidio}

El asesinato de mujeres en los conflictos armados cuenta con especificidades del contexto social, se encuentran relacionados con la violencia sexual y con mecanismos de terror ante las comunidades enemigas. En la República Democrática del Congo se utiliza la categoría «destrucción vaginal», que refiere al tipo de ataque contra las mujeres, en muchos casos, llevando a su muerte (Segato, 2014, p. 66). Existe también la denominada violencia aleccionadora, que se utiliza como mecanismo de coacción ante el desvío a la norma o la transgresión del rol de género —como es el caso de

\footnotetext{
${ }^{5}$ En concreto en Kivu del Norte, Kivu del Sur, Katanga y Distrito de Ituri En la parte oriental de la República Democrática del Congo se han denunciado unos 200.000 casos desde 1996; y en 2013 el gobierno registró 15.352 (Mesa, 2012). En concreto en Kivu del Norte, Kivu del Sur, Katanga y Distrito de Ituri.
} 
las agresiones a lideresas, mujeres que cumplen papeles de sujetas políticas, así como las violaciones a mujeres guerrilleras por parte de los ejércitos regulares-. Estas violencias se fijan en torno a la división sexual de los roles de género, mostrándose la violación como herramienta de humillación, ruptura y dominación del género masculino-militar sobre el femenino-civil, condicionando una violencia específica hacia las mujeres o todo aquello definido como femenino. El asesinato de mujeres en los conflictos armados está ligado a la impersonalidad del contexto, los cuales suelen darse con una intensa «escena bélica informal» a través de distintos grupos armados -legales e ilegales-, siendo conflictos internos que se adaptan a través de décadas (Segato, 2014).

En países donde existe algún conflicto armado, la cantidad de feminicidios ha aumentado, evidenciando una correlación entre guerra y feminicidios (véase gráfica 1).

Gráfica 1. Incremento de asesinatos en el marco de conflictos armados: Honduras, Guatemala y El Salvador.

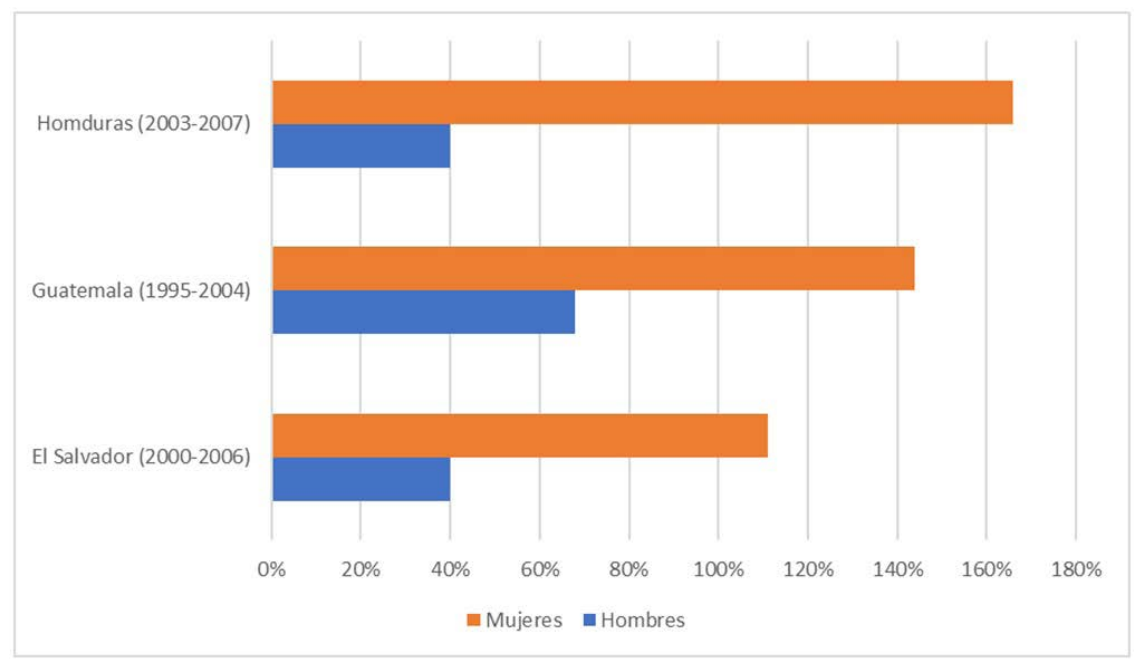

Fuente: elaboración propia a partir de PNUD (2013).

Las tasas de las mujeres se duplican, triplican y cuadriplican, observándose un aumento específico de estas en situaciones no solo de conflicto, sino también de «pacificación»(Carcedo, 2010, pp. 40-42). 


\section{Los roles de género en el conflicto: reificación y disidencia}

El discurso binario conceptualiza a las mujeres como «complementarias» de los hombres, relegándolas a una posición de objetos, sin intereses ni derechos iguales al de los hombres. De este modo, el sistema patriarcal se estructura en una jerarquía social en la que el género femenino se encuentra subordinado al masculino. En este sentido, las mujeres son objetivadas, constituyendo su ser social en torno a un cuerpo sin significado propio: como un ser-de-otros y como un cuerpo-para-otros. Dentro de esta estructura binaria el margen de actuación es limitado, los sujetos están constituidos de significantes culturales que se encuadran en el discurso hegemónico que inmoviliza las categorías de hombre y mujer.

Por consiguiente, las personas se encuentran en sistemas reificados de significados, careciendo del reconocimiento subjetivo al reducirse a elementos objetivados o mediante la autorreificación, cuando el sujeto considera que los elementos objetivados son elegidos (Honneth, 2007, 2010; Osborne, 2009). El género se plantea entonces como una construcción falsa y estática que establece un orden simbólico y que ignora la dimensión

[ 94 ] subjetiva (Lamas, 2003). La diferencia sexual actúa como un estructurante simbólico y psíquico, formando una relación de dominación masculina que aparece como «natural».

El imaginario social de la masculinidad hegemónica lleva a establecer la violencia como privilegio masculino, incluso como deber para clasificar como «hombre» a aquel ser sexuado como tal. La guerra establece así un vínculo con la masculinización — relacionada con la virilidad y la violencia (Pisano, 2001) - y el estereotipo de varón en la racionalidad, frialdad, fuerza, valentía y arrojo, contraponiéndose a los estereotipos asignados a la mujer de pasividad, indecisión, irracionalidad, sentimentalismo y debilidad (Magallón, 1998).

La representación de la mujer como alma dócil y pasiva se configura con respecto a la oposición a lo violento, relacionado con el hombre. Esta visión establece la antítesis entre el denominado «bello sexo» - la mujery el "soldado macho» - asociado con la valentía, la fuerza y el dominio del hombre- (Rojas, 1997). Este vínculo entre poder y violencia refuerza la construcción social sexuada, como la dicotomía entre mujer-pacífica y 
hombre-violento (Magallón, 1998), y se acentúan las asimetrías de género, tanto en la participación del conflicto como de quienes sufren los efectos.

Los hombres son visibilizados como héroes enfrentando al enemigo y las mujeres son concebidas como víctimas indirectas o como parejas afectivas de los actores armados, siempre desde un rol pasivo (Pino, 2004). De manera que la reificación en los conflictos armados se blinda ante la construcción patriarcal: las mujeres son observadas como víctimas y los hombres como perpetradores de la violencia. Esta construcción reificada deja al margen la posibilidad de analizar a las mujeres a partir de la posición de victimarias y los hombres como víctimas, opciones que se encuentran en las situaciones de guerra (Stemple, 2009).

\subsection{La reificación del género: las mujeres víctimas}

La etiqueta de "víctima» en los conflictos armados se establece para articular los procesos determinados por el sufrimiento de la violencia política, configurándose de diversas maneras. Existen distintos niveles de víctimas: aquellas que han sufrido la violencia a través de la pérdida de personas - normalmente, en relación con los hombres: huérfanas, viudas, madres que han perdido hijos(as) - y aquellas personas que han sufrido la violencia política en primera persona.

La naturalización de las categorías —ya sea víctima o mujer - implanta una identidad esencializada que se muestra como única y homogénea, produciendo una fijación en las cuales todas las personas que son víctimas se envuelven en la misma definición (Ahmed, 1996; Butler, 1993). Se establece un imaginario del sujeto víctima, articulando experiencias como una misma, homogeneizando la categoría de víctima en correspondencia de no-víctima, sesgando las distintas vivencias y realidades.

Se ha de tener en cuenta que las mujeres en el mundo - aun admitiendo diferencias culturales, económicas y sociales- son la parte subordinada, dominada y explotada dentro del sistema patriarcal, en su mayoría víctimas. En este sentido, en los conflictos armados las mujeres representan la mayoría de las víctimas vivas, las cuales son «cazadas como animales, convertidas en botín de guerra o en objeto de intercambio, las mujeres como colectivo han quedado sujetas a la violencia del grupo masculino» (Vendrell, 2013, p. 68). 
Esta limitación del concepto, así como de la presentación de los roles de género estereotipada, ofrece un análisis sesgado al no tener en cuenta la variedad de los roles existentes, ni los cambios de las funciones que afectan las relaciones de género (Bell y Narayanaswamy, 2003, p. 6). El discurso de protección a las mujeres debido a su «fragilidad» encubre un discurso sexista que interpreta la diferencia como jerarquía de una superioridad masculina, en la cual la mujer -así como los y las menores, o la población ancianase encuentran ubicados en la caracterización de población vulnerable, convirtiendo la diferencia en «guante de terciopelo sobre el puño de hierro de la dominación» (Mackinnon, 1995, p. 398).

La correlación directa entre mujeres y víctimas reifica la identidad de víctima con la de mujer. La construcción del imaginario del sujeto víctima se enraíza con la construcción del rol femenino, instituyendo la identificación con la vulnerabilidad de las mujeres como «femenino» y como víctima. Por lo tanto, se establece la posición de víctimas como «condición» en las mujeres y no como «situación». Esta propuesta conceptual tiene significativas consecuencias analíticas y políticas (Blair, 2012, p. 120). El posicionamiento de «condición» reifica a las mujeres en su rol de víctima, generando una posición estática e identitaria como mujer. Esta situación da lugar a la victimización de las mujeres, de modo que en este sistema patriarcal las mujeres se encuentran en «un juego perverso de amenazas y protección que las mantiene sujetas y dependientes» (Vendrell, 2013, p. 40).

Esta reificación del rol de femenino niega la posibilidad de atender otras realidades, limitando la agencia de las mujeres víctimas. Si bien es necesario - debido a la gran cantidad de mujeres víctimas- tener en cuenta la posición de las mujeres como categoría víctimas en el escenario bélico, estas no han de observarse como sujetas pasivas, "por lo que dar voz a quienes sufren y tratan de atajar ese sufrimiento no ha de impedirnos el oír y decir la vitalidad y la fuerza con la que tratan de seguir con sus vidas» (Magallón, 2006, p. 41).

Muchas organizaciones de mujeres - que han sufrido violencia tanto en contextos de conflicto armado como de paz- en el mundo se han resignificado aludiendo al concepto de «supervivientes». Este concepto rompe con la cosificación de las mujeres como víctimas y busca su empoderamiento. No obstante, el concepto es rechazado por una parte de las organizaciones de víctimas, debido a la concepción de este término, «en 
tanto que implica la existencia de un sujeto victimario, es decir, de alguien responsable de perpetuar la violencia» (Seminario Mujeres en Situación de Conflicto, 2008, p. 15).

\subsection{Disidencias de género: mujeres victimarias y sujetas políticas}

La progresiva participación de las mujeres como actoras de violencia o como sujetas políticas desmonta la reificación de las posturas de víctimamujer y hombre-victimario. Si bien la participación de las mujeres como victimarias no rompe con la ausencia universal de estas en los campos de batalla, la existencia de mujeres que participan activamente en la generación de la violencia ha de observarse como una realidad para tener en cuenta.

La participación de las mujeres en los grupos armados ilegales ha sido cada vez mayor, pero el proceso de incorporación de las fuerzas irregulares a los ejércitos regulares ha estado marcado por la ausencia de las mujeres, siendo excluidas de la esfera militar (Malesevic, 2010). Esta situación se identifica en algunos países como una menor brecha de género, donde se sigue contando con menor cantidad de mujeres en sus ejércitos. La única excepción a esta realidad es Israel, que cuenta con un servicio militar obligatorio tanto para mujeres como para hombres, pero responde a un caso particular.

Sean cuales sean los motivos que llevan a las mujeres a tomar las armas, estamos convencidos ahora de que también ellas pueden encerrar en sí mismas un potencial de agresividad que rompe con la imagen tradicional de la mujer [...] ahora sabemos que pueden participar en una guerra organizada o lanzar bombas con la misma determinación que los hombres (Badinter, 1987, p. 187).

Por tanto, las mujeres que han pertenecido a estructuras militares son disidentes de los roles femeninos tradicionales y de las expectativas que se tenían sobre ellas. Así pues, los numerosos ejemplos de mujeres como combatientes activas o sujetas políticas de los conflictos armados muestran que los estereotipos de género conllevan a una visión «miope o ingenua» (Bell y Narayanaswamy, 2003, p. 13). Al enfocar la mirada hacia la presencia de las mujeres como victimarias en los distintos conflictos armados se puede observar la magnitud de este fenómeno (véase mapa 1). 
Mapa 1. Países donde se conoce la participación de mujeres en grupos armados ilegales.

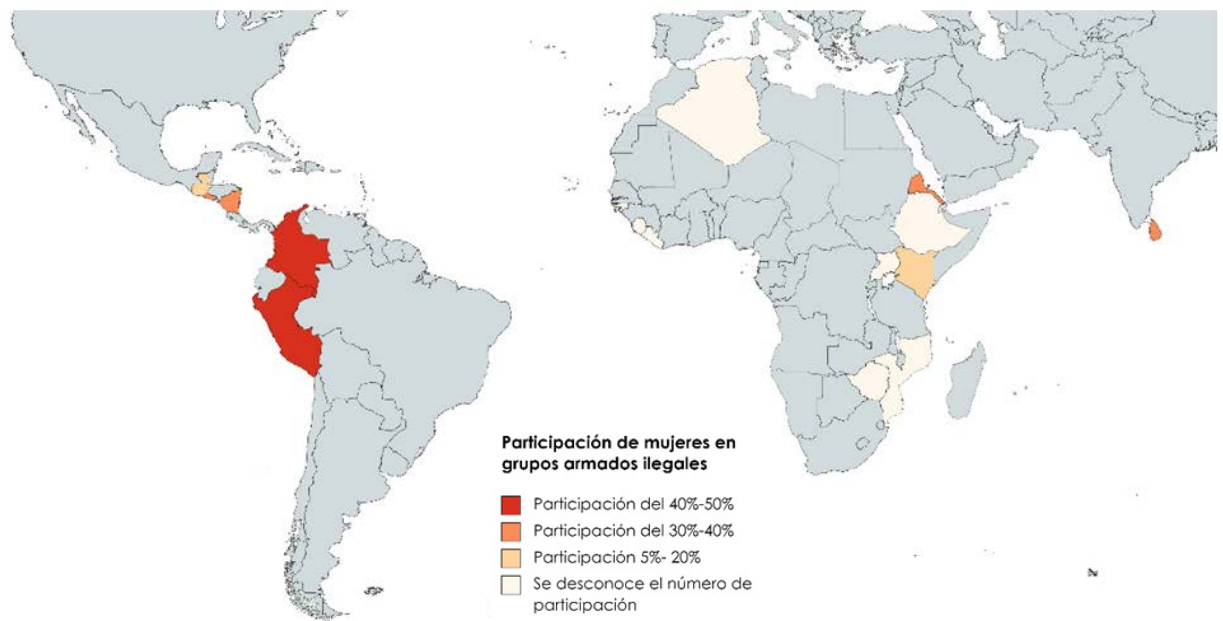

Fuente: elaboración propia a partir de Barth (2002); Arnfred (1988); Macdonald, Holden y Ardener (1987); Luciak (2001); Turriago y Bustamante (2003); Uricoechea (2000).

En Sri Lanka, 30\% de los Tigres Tamiles eran mujeres y, en igual [ 98 ] porcentaje, parte de los ejércitos revolucionarios en Nicaragua y Eritrea (Barth, 2002). En África, la presencia de mujeres combatientes en ejércitos guerrilleros y movimientos de liberación se conocen en Etiopía, Namibia, Zimbabwe, Mozambique, Argelia, Liberia, Sierra Leona, Guinea-Bissau, Uganda y Djibout (Barth, 2002; Arnfred, 1988). También fue significativa la participación de mujeres en las filas del Congreso Nacional Africano (ANC) o el $5 \%$ de participación en las guerrillas Mau-Mau de Kenia (Macdonald, Holden y Ardener, 1987).

En El Salvador las mujeres fueron 30\% de la población desmovilizada del Frente Farabundo Martí para la Liberación Nacional (FMLN), en Guatemala constituyeron $15 \%$ de la Unidad Revolucionaria Nacional Guatemalteca (URNG) y cerca de $25 \%$ en sus cuadros políticos. La presencia de mujeres en el Partido Comunista del Perú Sendero Luminoso (PCP-SL) es todavía más sorprendente, ya que se calcula que cerca de $40 \%$ de su militancia eran mujeres, conformando más de $50 \%$ de su Comité Central (Luciak, 2001).

En Colombia, entre $24 \%$ y $27 \%$ de las personas desmovilizadas tras los acuerdos de paz de las décadas de 1980 y 1990 eran mujeres, teniendo 
en cuenta que uno de los porcentajes más altos de mujeres se refiere al M-19 $(31,5 \%)$ y con menor proporción la CRS $(17,5 \%)$. La participación de las mujeres en las FARC-EP se encontraba en torno a 40\% en el 2000 (Turriago y Bustamante, 2003; Uricoechea, 2000).

Desde marzo de 2013 las mujeres kurdas se enrolaron en las Unidades Femeninas de Protección (YPJ) en su lucha contra el Dáesh —Estado Islámico-y por el Estado de Kurdistán. Si bien esta participación responde a la necesidad de activos en el frente, muchas mujeres abanderan la lucha de una sociedad más equitativa (RT en Español, 3 de julio de 2015).

Por otro lado, la participación de las mujeres en los conflictos se ha dado de diversas formas. Estas han ocupado diversos roles frente a la mirada patriarcal que aglutina a las mujeres como grupo vulnerable: sujetas pasivas y víctimas naturales. Gran parte de las organizaciones de mujeres en los países en conflicto están nutridas por mujeres víctimas que participan, crean y construyen sociedad, "[El] esfuerzo que las mujeres deben hacer para transformarse de víctimas en supervivientes de la guerra» (Cockburn, 2007, p. 47).

Uno de los grandes logros de las organizaciones feministas y de mujeres fue el reconocimiento, en la normativa internacional, de la violencia sexual como arma de guerra y los tribunales ad hoc (Mendian, 2008; Villellas, 2010). Prueba de ello ha sido la aprobación en el 2000 por parte del Consejo de Seguridad de Naciones Unidas de la Resolución 1325, y las posteriores resoluciones que la complementan, en la conformación de una agenda internacional de «mujeres, paz y seguridad», y que entre sus objetivos está la participación activa de las mujeres en los procesos de paz.

Por consiguiente, la participación de las mujeres como sujetas políticas o partícipes activas de grupos armados han mostrado la falacia ante las categorías reificadas en torno al género: «las mujeres han pasado a asumir la militancia activa como parte de su proyecto de vida y de sus actuaciones como sujetos políticos» (Herrera y Pertuz, 2014, p. 151).

\section{Conclusiones}

La ideologización del sistema patriarcal, sujeto al discurso binario, estructura y reifica los roles de género como estructurantes sociales, que si bien actúan en los escenarios de paz se refuerzan en los contextos de 
violencia. De modo que el patriarcado le es funcional a la organización sexuada de la guerra.

La organización social de la guerra excluye a las mujeres del campo de combate y las sitúa como víctimas de la violencia armada, reificándolas en un rol pasivo. Sin embargo, la participación de las mujeres desde un rol activo - como victimarias o sujetas políticas- muestra la ruptura a la norma patriarcal que identifica a las mujeres como población vulnerable.

Los tres problemas abordados en el artículo - i) estratificación del género: organización social de la violencia; ii) violencias específicas contra las mujeres en los conflictos armados; y iii) reificación de los roles de género: violencia implícita- evidencian una estructura social estratificada con carácter universal, en la cual las mujeres son excluidas del campo de combate, que configura una organización social que refuerza y acentúa la violencia contra las mujeres a través de la violencia explícita en sus cuerpos y revictimizadas en la condicionalidad del género al rol de víctima.

No obstante, aun existiendo estratificación de género en la guerra $-\mathrm{y}$ en las negociaciones de paz-, las mujeres han participado de diversas formas

[100] generando una ruptura con el relato binario hegemónico. La participación equitativa de las mujeres en los espacios políticos de decisión, así como la atención de las mujeres como sujetas políticas en el análisis y la acción, devienen como hechos irresolubles para un verdadero pacto democrático.

\section{Referencias bibliográficas}

1. Aafjes, Astrid. (1998). Gender Violence: The Hidden War Crime. Washington, D. C: Women, Law and Development International.

2. Ahmed, Sara. (1996). Moving Spaces. Black Feminism and PostColonial Theory. Theory, Culture and Society, 13, pp. 139-146. https://doi. org/10.1177/026327696013001008

3. Arnfred, Signe. (1988). Women in Mozambique: Gender Struggle and Gender Politics. Review of African Political Economy, 15 (41), pp. 5-16. https://doi. org/10.1080/03056248808703759

4. Badinter, Elizabeth. (1987). El uno es el otro. Bogotá, D. C.: Planeta.

5. Barth, Elise F. (2002). Peace as Disappointment. The Reintegration of Female Soldiers in Pots-Conflicts Societies: A Comparative Study from Africa. International Peace Research Institute. https://files.prio.org/Publication_files/Prio/Barth\%20 (2002)\%20Peace\% 20as\%20Disappointment\%20(PRIO\%20Report\%203-2002).pdf 
6. Bell, Emma y Narayanaswamy, Lata. (2003). Género y conflictos armados. PNUD. http://americalatinagenera.org/newsite/images/cdr-documents/publicaciones/ doc_238_generoyconfarmados2.pdf

7. Blair, Elsa. (2012). Un itinerario de investigación sobre la violencia. Contribución a una sociología de la ciencia. Medellín: Universidad de Antioquia.

8. Bocchetti, Andrea. (1996). Lo que quiere una mujer. Madrid: Cátedra.

9. Brownmiller, Susan. (1975). Against Our Will. Men, Women and Rape. New York: Bantam.

10. Butler, Judith. (1993). Cuerpos que importan. Buenos Aires: Paidós.

11. Byrne, Bridget. (1996). Gender, Conflict and Development. Volume I. Brighton: Institute of Development Studies.

12. Carcedo, Ana. (2010). No olvidamos niaceptamos: femicidio en Centroamérica 2000-2006. San José de Costa Rica: Cefemina y Horizons.

13. Cockburn, Cynthia. (1998). The Space Between Us: Negotiating Gender and National Identities in Conflict. London: Zed.

14. Cockburn, Cynthia. (2007). Mujeres ante la guerra. Barcelona: Icaria.

15. Connell, Raewyn W. (1995) Masculinities. Cambridge: Cambridge Polity.

16. Coomaraswamy, Ananda K. (1999). El tiempo y la eternidad. Barcelona: Kairós.

17. Dador, Jennie. (2005). Impunidad, pongámosle fin. Violencia sexual contra las mujeres en conflicto armado y postconflicto en América Latina. Universidad Autónoma de Barcelona. https://www.bib.uab.cat/socials/exposicions/donesvio/docs/ conflictes/americalatina.pdf

18. De Waal, Frans. (2005). Our Inner Ape: A Leading Primatologist Explains Why We Are Who We Are. New York: Riverhead.

19. Durkheim, Emile. (1986). The Concept of the State. In: Giddens, Anthony (Ed.) Durkheim on the Politics and the State (pp. 32-72). Cambridge: Cambridge Polity.

20. Fisas, Vicenç. (1987). Introducción al estudio de la Paz y de los Conflictos. Barcelona: Lerna.

21. Foucault, Michel. (1975). Vigilar y Castigar. Madrid: Siglo Xxı.

22. Fry, Douglas. (2007). Beyond War: The Human Potential for Peace. Oxford: Oxford University.

23. Giles, Wenona \& Hyndman, Jennifer. (2004). Sites of Violence. Gender and Conflict Zones. California: University of California. https://doi.org/10.1525/ california/9780520230729.001.0001

24. Goldstein, Joshua S. (2001). War and Gender: How Gender Shapes the War System and Vice Versa. Cambridge: Cambridge University.

25. Gutman, Roy y Rieff, David (dirs.) (2003). Crímenes de Guerra. Barcelona: Random House Mondadori. 
26. Herrera Martha Cecilia y Pertuz, Carol. (2014). Narrativas femeninas del conflicto armado y la violencia política en Colombia: contar para rehacerse. Revista de Estudios Sociales, 53, pp. 150-162. https://doi.org/10.7440/res53.2015.12

27. Homero. (1996). La Iliada. Madrid: Alba.

28. Honneth, Axel. (2007). Reificación. Un estudio en la teoría del reconocimiento. Buenos Aires: Katz.

29. Honneth, Axel. (2010). Reconocimiento y menosprecio Sobre la fundamentación normativa de una teoría social. Buenos Aires: Katz.

30. Kaldor, Mary. (1999). New and Old Wars: Organized Violence in a Global Era. Stanford: Stanford University.

31. Kandiyoti, Deniz. (2000). Guest Editor's Introduction. The Awkward Relationship: Gender and Nationalism. Nations and Nationalism, 6 (4), pp. 491-494. https://doi.org/10.1111/j.1354-5078.2000.00491.x

32. Lagarde, Marcela. (2006). Introducción. Por la vida y la libertad de las mujeres, fin al feminicidio. En: Russell, Diana y Harmes, Roberta (eds). Feminicidio: una perspectiva global (pp. 15-42). México, D. F.: UNAM.

33. Lamas, Marta. (2003). Género. Claridad y Complejidad. PNUD. http:// americalatinagenera.org/newsite/images/cdr-documents/publicaciones/doc_202 genero-claridad-complejidad.pdf

34. Liss, Sarah. (2004, mayo 13). Bell Hooks. African-American Feminist Icon Fights Fascism with Love. Now Toronto. https://nowtoronto.com/news/bell-hooks

35. Luciak, Ilja. (2001). Alter the Revolution. Gender and Democracy in El Salvador, Nicaragua and Guatemala. Baltimore: The Johns Hopkins University.

36. Macdonald, Sharon; Holden, Shirley \& Ardener, Patt. (1987). Images of Women in Peace \& War: Cross Cultural \& Historical Perspectives. London: Macmillan. https://doi.org/10.1007/978-1-349-18894-9

37. Mackenzie, Megan. (2010). Securitizing Sex? Towards a theory of the utility of wartime sexual violence. International Feminist Journal of Politics, 12 (2), pp. 202221. https://doi.org/10.1080/14616741003665250

38. Mackinnon, Catharine A. (1995). Hacia una teoría feminista del Estado. Madrid: Cátedra.

39. Magallón, Carmen. (1998). Sostener la vida, producir la muerte: estereotipos de género y violencia. En: Fisas, Vicent (ed.). El sexo de la violencia. Género y cultura de la violencia (pp. 93-116). Barcelona: Icaria.

40. Magallón, Carmen. (2006). Mujeres en pie de paz. Madrid: Siglo Xxı.

41. Malesevic, Sinisa. (2010). The Sociology of War and Violence. Cambridge: Cambridge University. https://doi.org/10.1017/CBO9780511777752

42. Malesevic, Sinisa. (2020). El auge de la brutalidad organizada. Una sociología histórica de la violencia. Valencia: Universitat de València. 
43. Mann, Michael. (1986). The Sources of Social Power I: A History of Power from the Beginning to $A D$ 1760. Cambridge: Cambridge University. https://doi. org/10.1017/CBO9780511570896

44. Mendian, Irantxu. (2008). Aportes sobre el activismo de las mujeres por la paz. Cuadernos de Trabajo Hegoa, 48. https://ojs.ehu.eus/index.php/hegoa/article/ download/10601/9841

45. Mesa, Manuela. (2012). Prevención de conflictos, paz y seguridad: Avances conceptuales, experiencias y lecciones aprendidas. Pensamiento Propio, 36/37, pp. 189-219.

46. Naciones Unidas. Consejo de Seguridad. Resolución 1325/2000. (31 de octubre de 2000). https://www.un.org/womenwatch/ods/S-RES-1325(2000)-S.pdf

47. Olujic, Maria B. (1998). Embodiment of Terror: Gendered Violence in Peacetime and Wartime in Croatia and Bosnia-Herzegovina. Medical Anthropology Quarterly, 12 (1), pp. 31-50. https://doi.org/10.1525/maq.1998.12.1.31

48. Organization of African Unity. (2000). Rwanda, the Preventable Genocide. International Panel of Eminent Personalities. REFWORLD. https://www.refworld.org/ pdfid/4d1da8752.pdf

49. Osborne, Raquel. (2009). Apuntes sobre violencia de género. Barcelona: Bellaterra.

50. Physicians for Human Rights. (2002). War-Related Sexual Violence in Sierra Leone. Massachusetts: PHR) \& UN Sierra Leone.

51. Pinker, Steven (2012). Los ángeles que Ilevamos dentro. El declive de la violencia y sus implicaciones. Barcelona: Paidós.

52. Pino, Ana Cristina. (2004). El patriarcado y sus manifestaciones en el conflicto armado. Bogotá, D. C.: AHIGE-REPEM.

53. Pisano, Margarita. (2001). El triunfo de la masculinidad. Santiago de Chile: Surada.

54. Programa de las Naciones Unidas para el Desarrollo (PNUD). (2003). El conflicto, callejón con salida. Informe Nacional de Desarrollo Humano para Colombia-2003. Bogotá, D. C.: PNUD.

55. Programa de las Naciones Unidas para el Desarrollo (PNUD). (2013). Seguridad ciudadana con rostro humano: diagnóstico y propuestas para América Latina. Informe Regional de Desarrollo Humano 2013-2014. Nueva York: PNUD.

56. Rehn, Elisabeth y Johnson Sirleaf, Ellen. (2002). Mujeres, guerra y paz. Informe sobre el Progreso de las Mujeres en el Mundo, Vol ı. Nueva York: UNIFEM.

57. Rojas, María Cristina. (1997). Las almas bellas y los guerreros justos. Revista Javeriana. 128 (639), pp. 351-159.

58. RT en Español (3 de julio de 2015). Mujeres kurdas: en guerra contra el ISIS - Documental de RT [Video]. Youtube. https://www.youtube.com/ watch?v=q5GUEQmZ_gM 
59. Russell, Diana y Radford, Jill (eds.). (2006). Feminicidio. La política del asesinato de las mujeres. México, D. F.: UNAM.

60. Segato, Rita Laura. (2014). Las nuevas formas de la guerra y el cuerpo de las mujeres, Sociedade e Estado, 29 (2), pp. 341-371. https://doi.org/10.1590/S010269922014000200003

61. Seminario Mujeres en Situación de Conflicto. (2008). Mujeres en situaciones de conflicto: reflexiones en clave feminista. Madrid: Hegoa y ACSUR Las Segovias.

62. Service, Elman R. (1978). Profiles in Ethnology. New York: Haper and Row.

63. Stemple, Lara. (2009). Male Rape and Human Rights. Hastings Law Journal, 60 (3), pp. 605-646.

64. Stockholm International Peace Research (SIPRI). (2008). SIPRI Yearbook 2008. Oxford: Oxford University.

65. Tilly, Charles. (1985). War Making and State Making as Organized Crime. In: Evans, Peter; Rueschemeyer, Dietrich \& Skocpol, Theda (Eds.). Bringing the State Back In (pp. 169-191). New York: Cambridge University. https://doi.org/10.1017/ CBO9780511628283.008

66. Tilly, Charles. (1990). Coerción, capital y los Estados europeos 990-1990. Madrid: Alianza.

67. Turriago, Gabriel y Bustamante, José María. (2003). Estudio de los procesos de reinserción en Colombia 1991-1998. Bogotá, D. C.: FIP.

68. Uricoechea, Fernando. (2000). Perspectiva institucional de los procesos de [104 ] reinserción. En: Ministerio del Interior. De las armas a la democracia, tomo II. Bogotá: Ministerio del Interior.

69. Vendrell, Joan. (2013). La violencia del género. Una aproximación desde la antropología. México, D. F.: UAEM.

70.Villellas, María. (2010). La violencia sexual como arma de guerra. Quaderns de Construcció de Pau, 15). https://escolapau.uab.cat/img/qcp/violencia_sexual_ guerra.pdf

71. Weber, Max. (1964). Economía y Sociedad. Madrid: FCE. 